\title{
Characterization of the K2-18 multi-planetary system with HARPS ${ }^{\star}$ A habitable zone super-Earth and discovery of a second, warm super-Earth on a non-coplanar orbit
}

\author{
R. Cloutier1, 2,3, N. Astudillo-Defru ${ }^{4}$, R. Doyon ${ }^{3}$, X. Bonfils ${ }^{5}$, J.-M. Almenara ${ }^{4}$, B. Benneke ${ }^{3}$, F. Bouchy ${ }^{4}$,

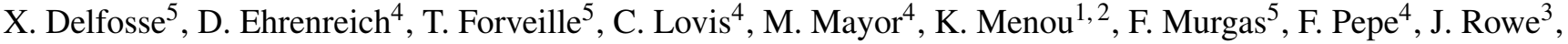 \\ N. C. Santos ${ }^{6,7}$, S. Udry ${ }^{4}$, and A. Wünsche ${ }^{5}$ \\ ${ }^{1}$ Dept. of Astronomy \& Astrophysics, University of Toronto, 50 St. George Street, M5S 3H4, Toronto, ON, Canada \\ e-mail: cloutier@astro.utoronto.ca \\ ${ }^{2}$ Centre for Planetary Sciences, Dept. of Physical \& Environmental Sciences, University of Toronto Scarborough, 1265 Military \\ Trail, M1C 1A4, Toronto, ON, Canada \\ ${ }^{3}$ Institut de Recherche sur les Exoplanètes, Département de physique, Université de Montréal, CP 6128 Succ. Centre-ville, H3C 3J7, \\ Montréal, QC, Canada \\ 4 Observatoire Astronomique de l'Université de Genève, 51 chemin des Maillettes, 1290 Versoix, Switzerland \\ 5 Université Grenoble Alpes, CNRS, IPAG, 38000 Grenoble, France \\ 6 Instituto de Astrofísica e Ciências do Espaço, Universidade do Porto, CAUP, Rua das Estrelas, 4150-762 Porto, Portugal \\ 7 Departamento de Física e Astronomia, Faculdade de Ciências, Universidade do Porto, Rua do Campo Alegre, 4169-007 Porto, \\ Portugal
}

Received 12 July 2017 / Accepted 6 September 2017

\begin{abstract}
Aims. The bright M2.5 dwarf K2-18 $\left(M_{\mathrm{S}}=0.36 M_{\odot}, R_{\mathrm{S}}=0.41 R_{\odot}\right)$ at $34 \mathrm{pc}$ is known to host a transiting super-Earth-sized planet orbiting within the star's habitable zone; K2-18b. Given the superlative nature of this system for studying an exoplanetary atmosphere receiving similar levels of insolation as the Earth, we aim to characterize the planet's mass which is required to interpret atmospheric properties and infer the planet's bulk composition.

Methods. We have obtained precision radial velocity measurements with the HARPS spectrograph. We then coupled those measurements with the $\mathrm{K} 2$ photometry to jointly model the observed radial velocity variation with planetary signals and a correlated stellar activity model based on Gaussian process regression.

Results. We measured the mass of K2-18b to be $8.0 \pm 1.9 M_{\oplus}$ with a bulk density of $3.3 \pm 1.2 \mathrm{~g} / \mathrm{cm}^{3}$ which may correspond to a predominantly rocky planet with a significant gaseous envelope or an ocean planet with a water mass fraction $\gtrsim 50 \%$. We also find strong evidence for a second, warm super-Earth K2-18c $\left(m_{\mathrm{p}, \mathrm{c}} \sin i_{\mathrm{c}}=7.5 \pm 1.3 M_{\oplus}\right)$ at approximately nine days with a semi-major axis $\sim 2.4$ times smaller than the transiting K2-18b. After re-analyzing the available light curves of K2-18 we conclude that K2-18c is not detected in transit and therefore likely has an orbit that is non-coplanar with the orbit of K2-18b although only a small mutual inclination is required for $\mathrm{K} 2-18 \mathrm{c}$ to miss a transiting configuration; $|\Delta i| \sim 1-2^{\circ}$. A suite of dynamical integrations are performed to numerically confirm the system's dynamical stability. By varying the simulated orbital eccentricities of the two planets, dynamical stability constraints are used as an additional prior on each planet's eccentricity posterior from which we constrain $e_{\mathrm{b}}<0.43$ and $e_{\mathrm{c}}<0.47$ at the level of $99 \%$ confidence.

Conclusions. The discovery of the inner planet K2-18c further emphasizes the prevalence of multi-planet systems around M dwarfs. The characterization of the density of K2-18b reveals that the planet likely has a thick gaseous envelope which, along with its proximity to the solar system, makes the K2-18 planetary system an interesting target for the atmospheric study of an exoplanet receiving Earth-like insolation.
\end{abstract}

Key words. techniques: radial velocities - methods: statistical - planets and satellites: detection planets and satellites: fundamental parameters - planets and satellites: individual: K2-18

\section{Introduction}

Exoplanets orbiting within their host star's habitable zone may have surface temperatures that allow for the presence of liquid water on their surfaces, depending on the properties of the planetary atmosphere (Kasting et al. 1993). The presence of liquid water is a condition likely required to sustain extraterrestrial life.

\footnotetext{
* Table A.2 is also available at the CDS via anonymous ftp to cdsarc.u-strasbg. fr (130.79.128.5) or via http://cdsarc.u-strasbg.fr/viz-bin/qcat?J/A+A/608/A35
}

This implies that habitable zone exoplanets receive comparable levels of stellar insolation to what the Earth receives from the Sun. Habitable zone exoplanets therefore represent superlative opportunities to search for life outside of the solar system via the characterization of their atmospheric structure and composition via transmission spectroscopy for transiting exoplanets.

M dwarf host stars are ideal targets to probe potentially habitable exoplanetary atmospheres (e.g. Kaltenegger et al. 2011; Rodler \& López-Morales 2014). Transmission spectroscopy observations of transiting habitable zone (HZ) exoplanets around 
M dwarfs are favourable compared to those around Sun-like stars given the increased depth of the transit for a planet of a given size (e.g. Stevenson et al. 2010; Kreidberg et al. 2014). In addition, the orbital periods corresponding to the $\mathrm{HZ}$ are less than those around Sun-like stars (weeks for those in the HZ of M dwarfs, compared to 12 months) thus increasing the number of accessible transit events within a given observational baseline. $\mathrm{M}$ dwarfs are also known to frequently host multiple small planets (typically 2.5 planets per star with $0.5 \leq r_{\mathrm{p}} / R_{\oplus} \leq 4$ and within 200 days; Dressing \& Charbonneau 2015; Gaidos et al. 2016) thus enabling direct comparative planetology to be conducted on known multi-planet systems.

Montet et al. (2015) reported the detection of the HZ superEarth K2-18b originally proposed in the K2 light curve analysis of Foreman-Mackey et al. (2015). In these studies, two transit events were observed in Campaign 1 data from the re-purposed Kepler spacecraft mission $\mathrm{K} 2$ whose field coverage only lasted for $\sim 80$ days. The existence of the planet was confirmed and uncertainties regarding its ephemeris were significantly reduced in Benneke et al. (2017; hereafter B17) who used follow-up transit observations with the Spitzer Space Telescope to detect an additional transit event. The now confirmed super-Earth K2-18b orbits an M2.5 dwarf with a period of 32.9 days placing it directly within the star's habitable zone (Kopparapu et al. 2013). The measured radius of $2.38 R_{\oplus}$ is suggestive of an extended H/He envelope (Valencia et al. 2013; Rogers 2015; Fulton et al. 2017) that may contain additional molecular species such as water and/or methane that could be detectable with the James Webb Space Telescope (JWST; Beichman et al. 2014). Owing to the proximity of the system $(\sim 34 \mathrm{pc}, V=13.5, I=11.7, K=8.9$; Cutri et al. 2003; Zacharias et al. 2013), K2-18 is truly an attractive target for characterizing the atmosphere of a HZ super-Earth with unprecedented precision in the JWST-era.

In this study we report the first measurement of the planetary mass of K2-18b using precision radial velocity measurements taken with the HARPS spectrograph. In this data we also find strong evidence for an additional planet of similar minimum mass whose orbit is interior to $\mathrm{K} 2-18 \mathrm{~b}$ but is not found to transit. In Sect. 2 we summarize the HARPS spectroscopic and K2 photometric observations used in our analysis, in Sect. 3 we analyze the periodic signals in the spectroscopic data and in Sect. 4 we discuss our various radial velocity modelling procedures. In Sect. 5 we present the results of our radial velocity analysis including the detection of a second super-Earth K2-18c in the system which we show is non-transiting and therefore is not perfectly coplanar with K2-18b in Sect. 6. Lastly we perform a dynamical analysis of the two-planet system in Sect. 7 to dynamically constrain the orbital eccentricities of the planets before concluding with a discussion in Sect. 8.

\section{Observations}

\subsection{HARPS spectra}

From April $2015 \quad(\mathrm{BJD}=2457117.5)$ to May 2017 $(\mathrm{BJD}=2457875.5)$, we collected 75 spectra of K2-18 (EPIC 201912552) with the high-resolution $(R=115000)$ HARPS spectrograph (Mayor et al. 2003; Pepe et al. 2004). The majority of exposure times were fixed to $1800 \mathrm{~s}$ with the exception of the following six epochs whose exposure times were modified to the following reported values: $2400 \mathrm{~s}(\mathrm{BJD}-2450000=7199.503915,7200.503114), 1200 \mathrm{~s}$ $($ BJD-2 $450000=7204.491167)$, and $900 \mathrm{~s}(\mathrm{BJD}-2450000=$ 7810.806284, 7814.760772, 7815.759421). The online HARPS pipeline returned the extracted, wavelength-calibrated spectra (Lovis \& Pepe 2007). Initial radial velocity estimates were computed from the cross-correlation of each spectrum with a numerical mask (Baranne et al. 1996; Pepe et al. 2002). Using each spectrum's initial estimate, all spectra were shifted to a common reference frame by their corresponding barycentric correction such that spectral features originating from the target star become aligned while telluric features are shifted by minus the epoch's barycentric correction. The median combination of these shifted spectra was then used to construct a custom reference spectrum at high signal-to-noise $(\mathrm{S} / \mathrm{N})$. A telluric template was then constructed from the median combination of all residual spectra after removal of the high $\mathrm{S} / \mathrm{N}$ reference stellar spectrum. The process of computing the median reference stellar spectrum was then repeated using the individual spectra with tellurics masked by the median telluric spectrum. We then computed precision radial velocities by performing a $\chi^{2}$-minimization of each spectrum with the reference spectrum (Astudillo-Defru et al. 2015). Radial velocity uncertainties were then estimated directly on the reference spectrum (Bouchy et al. 2001).

From the extracted spectra we also derived a number of activity indicators including the time series of the $\mathrm{H} \alpha$ index which is sensitive to chromospheric activity and is computed following the definition in Bonfils et al. (2007). For the M dwarf K2-18 ( $V=13.5$; Henden \& Munari 2014) the $\mathrm{H} \alpha$ index is favoured over the Ca II H+K Mt. Wilson S index (Wilson 1968; Baliunas et al. 1995) due to the low S/N obtained in the blue. From the $\mathrm{S}$ index we derived $\log R_{\mathrm{HK}}^{\prime}=-5.247 \pm 0.318$ (Astudillo-Defru et al. 2017). Additionally we derived the full width at half maximum (FWHM) and bi-sector inverse slope (BIS) shape parameters of the cross-correlation function which are modified by dark and/or bright active regions traversing the visible stellar surface. In Sect. 3 we use these ancillary time series to learn about the star's activity simultaneously with our radial velocity measurements. All spectroscopic time series are reported in Table A.2.

\subsection{K2 photometry}

K2-18 was observed in long-cadence mode during Campaign 1 of the K2 mission as part of the "Targeting M dwarfs with K2" proposal (GO1053 ${ }^{1}$, PI: B. Montet). The baseline of the K2 light curve is just 80 days but provides nearly continuous coverage between June 1st, $2014(\mathrm{BJD}=2456810.5)$ and August 20th, 2014 (BJD = 2456890.5$)$.

We obtained the full de-trended light curve from the MAST ${ }^{2}$ data retrieval service. As a result of the loss of two reaction wheels on-board the Kepler spacecraft, photometric observations from the $\mathrm{K} 2$ mission exhibit a reduced pointing precision, and hence photometric precision, compared to the original Kepler mission. Raw K2 light curves must be de-trended with the variable pointing of the spacecraft throughout the observing sequence. We selected the EVEREST reduction of the K2 light curve which performs this de-trending correction (Luger et al. 2016).

The majority of the residual photometric variability following de-trending of the light curve can be attributed to the intrinsic photometric variability of the star and two observed transits of K2-18b from Montet et al. (2015). Removal of the transit events provides a dataset that can be used to investigate the correlated

$1 \mathrm{~K} 2-18$ was also targeted in the following programmes: G01006, GO1036, GO1050, GO1051, GO1052, GO1059, GO1063, GO1075.

2 http://archive.stsci.edu/k2/hlsp/everest/search.php 


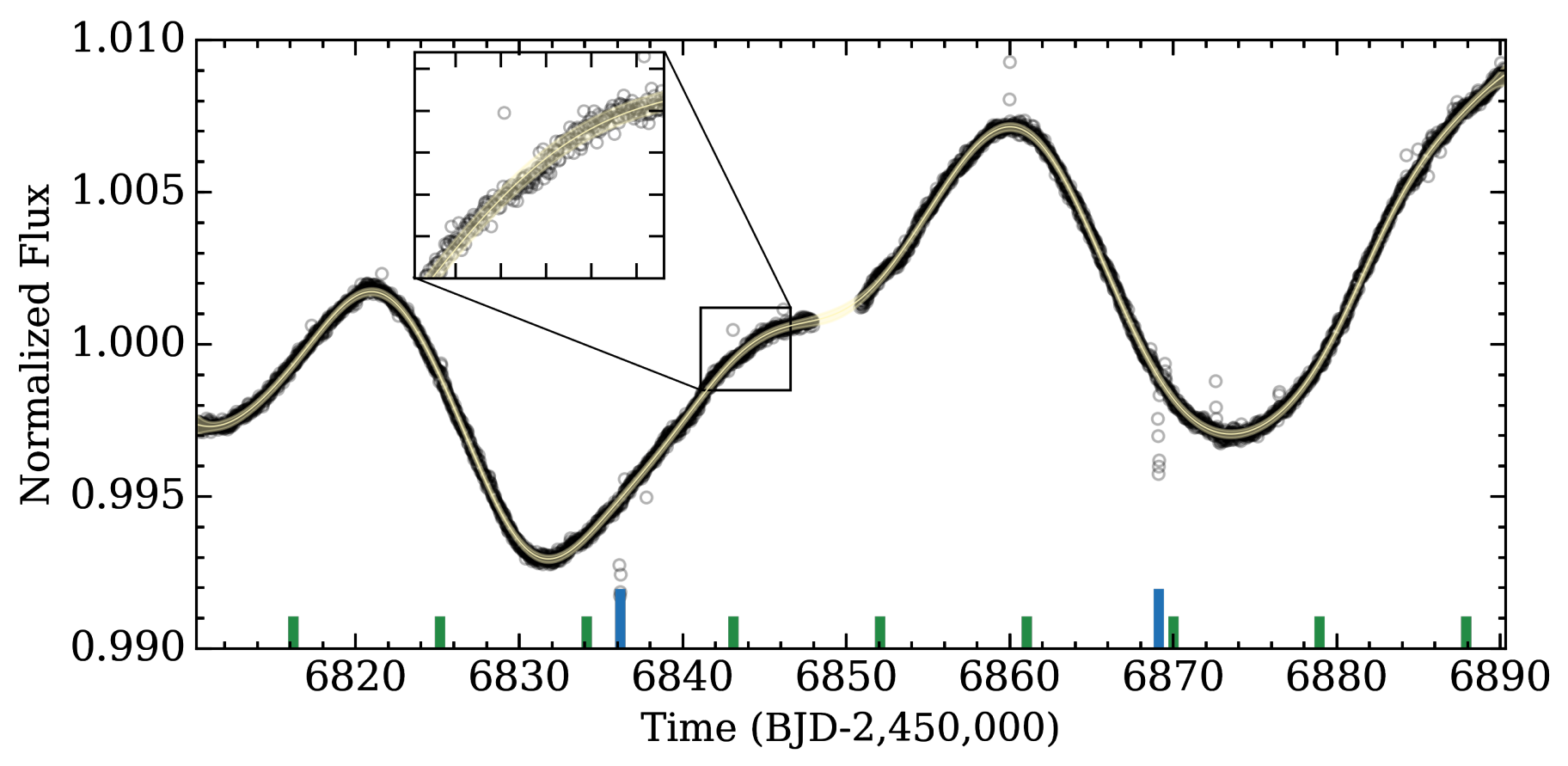

Fig. 1. K2 photometric light curve after the removal of known unphysical spurious signals. The two [transinsț blue ticks with the expected times of mid-transit for K2-18c highlighted with short green ticks (see Sect. 5). The isolid yellow curve? is the mean of the predictive GP distribution and the surrounding shaded regions mark its $99 \%$ confidence intervals. The upper left sub-panel is a magnified view of the highlighted region to aid in the visualization of the data and the GP fit.

photometric activity resulting from active regions traversing the visible stellar surface thus giving rise to the star's observed photometric variability. For reference, the de-trended light curve is shown in Fig. 1 along with our Gaussian process fit to the light curve (see Sect. 4.1 for an explanation of the fit).

\section{Periodogram analysis}

Accurate modelling of the stellar radial velocity (RV) variations requires knowledge of the strong periodicities present in the data. These signals include contributions from both orbiting planets and from the rotation of active regions present on the stellar surface which give rise to a correlated stellar RV signal which is modulated by the stellar rotation period and/or its harmonics. In the top panel of Fig. 2 we plot the Lomb-Scargle periodogram (Scargle 1982) of the raw RVs to determine which periodicities are present at high significance, that is, those with a low false alarm probability (FAP). In all LS-periodograms we calculated FAPs via bootstrapping with replacement using $10^{4}$ iterations and individually normalize each periodogram's power by its standard deviation.

Two important features are detected in the LS-periodogram of the RVs. The first is a forest of peaks ranging from $\sim 25-$ 45 days with distinct peaks centred on both the orbital period of $\mathrm{K} 2-18 \mathrm{~b}\left(P_{\mathrm{b}} \sim 33\right.$ days B17; FAP $\left.=2.9 \%\right)$ and the approximate stellar rotation period from the K2 photometry (see Sect. 4.1 for fitting of the stellar rotation period $P_{\text {rot }} \sim 38.6$ days; FAP $=$ $0.1 \%$ ). The second important feature is a pair of closely spaced peaks at $\sim 9$ days (FAP $<0.01 \%$ ) which because of their similar period and power likely result from a single source. This feature at approximately nine days constitutes the strongest periodic signal in the periodogram of the raw RVs and is not observed in the periodograms of any of the ancillary time series, nor in the periodogram of the window function, all of which are shown in the remaining panels of Fig. 2. The aforementioned time series include the K2 photometry (see Fig. 1), the window function or time sampling of the HARPS observations, and four spectroscopic activity indicators: the $\mathrm{S}$ index, $\mathrm{H} \alpha$ index, FWHM, and the BIS of the cross-correlation function. Together the presence of the strong $\sim 9 \mathrm{~d}$. signal in radial velocity and its absence elsewhere provides strong initial evidence for a second planet in the K2-18 system at $\sim 9 \mathrm{~d}$.

\section{Joint modelling of planets and correlated RV activity}

\subsection{Training the GP activity model on ancillary time series}

The K2 photometry of K2-18 exhibits quasi-periodic photometric variability with a semi-amplitude of $\sim 0.008$ mag and a rotation period of $P_{\text {rot }} \sim 38.6$ days as seen in Fig. 1. This makes K2-18 a moderately active early $\mathrm{M}$ dwarf in terms of its photometric variability (Newton et al. 2016) the origin of which likely results from the rotation of active regions across the projected stellar disk at or close to $P_{\text {rot }}$ owing to the characteristically low amplitudes of differential rotation in $\mathrm{M}$ dwarfs (Kitchatinov \& Olemskoy 2011). The observed photometric variability - or photometric activity - has a correlated manifestation in the variation of the star's apparent radial velocity and certain spectroscopic indicators because it is a single physical process that is responsible for the activity in each time series (Aigrain et al. 2012).

In order to obtain accurate and self-consistent detections of the planetary signals in radial velocity we must model the RV activity signal of K2-18 simultaneously with our planet model. The photometric stellar rotation period of $\sim 38$ days (see second panel in Fig. 2) is marginally detected in the LS-periodogram of the RVs. However $P_{\text {rot }}$ is clearly discernible by eye in the K2 light curve (Fig. 1) and has significant power in the LS-periodogram of the K2 light curve (second panel in Fig. 2) although the power is spread over a wide range of periodicities. Because of this we 


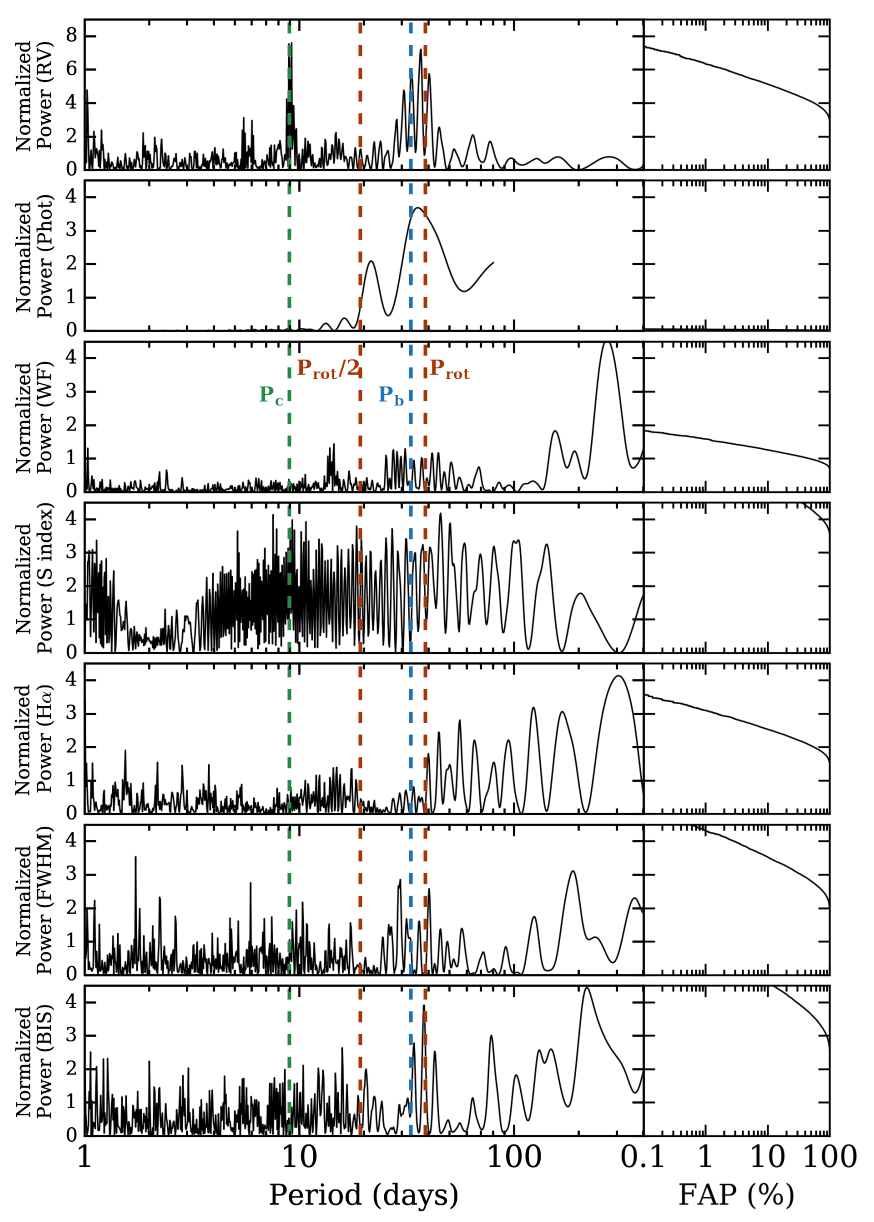

Fig. 2. Left column (top to bottom): Lomb-Scargle periodograms of the raw radial velocities (RV), the K2 photometry (Phot), the HARPS window function (WF), $\mathrm{S}$ index, $\mathrm{H} \alpha$, full width half maximum (FWHM), and bi-sector inverse slope (BIS) time series. The orbital periods of $\mathrm{K} 2-18 \mathrm{~b}, \mathrm{~K} 2-18 \mathrm{c}$, the stellar rotation period, and its first harmonic are

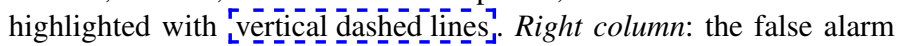
probability as a function of normalized periodogram power for each time series. The FAP curve for the photometry spans very low power and is barely discernible in its subpanel. Suffice it to say that any signal with normalized power $>10^{-2}$ has a FAP $\ll 0.1 \%$. The FAP curve for the $S$ index exhibits FAP $\gtrsim 30 \%$ for all power visible on its ordinate and therefore is only visible in the upper right of its subpanel.

consider in our first model - called Model 1 - the K2 photometric light curve, less the observed transits of $\mathrm{K} 2-18 \mathrm{~b}$, to train our RV activity model whose covariance properties are common with the observed photometric variability. However two important caveats had to be considered when adopting the K2 photometry as our training set. The first being that the baseline of the photometry spans just 80 days implying that any temporal variation whose characteristic timescale is greater than this baseline will remain unconstrained or at best weakly constrained. Secondly the K2 photometry were obtained nearly eight months prior to our HARPS observations such that any evolution in the covariance structure of the stellar activity between observing sequences from say magnetic activity cycles, would not be captured in the training set. For these reasons we also considered the BIS time series as an alternative training set in a second round of modelling called Model 2. Being contemporaneous with the $\mathrm{RV}$ measurements, training on the BIS time series mitigates the two aforementioned issues. In place of the BIS we also tested training on the $\mathrm{S}$ index, $\mathrm{H} \alpha$, and FWHM time series but find results consistent with training on the BIS. Following Faria et al. (2016) we also considered a joint activity + planet model but neglected any training of the activity model's covariance structure in a third model; Model 3. Finally for comparison purposes we also considered a fourth model - called Model 4 - that neglected any contribution from stellar activity.

To implement this joint modelling procedure we followed Cloutier et al. (2017) by using a Gaussian process (GP) regression model to model the covariance between adjacent observations in our training sets where applicable (i.e. in Models 1 and 2). GP regression is an attractive method for modelling the stochastic processes that gives rise to observable RV activity signals as it is non-parametric and therefore independent of an assumed functional form of the signal. The GP prior was represented by a multi-variate Gaussian distribution of functions described by a covariance matrix $K_{i j}=k_{i j}(\boldsymbol{\theta})+\sigma_{i}^{2} \delta_{i j}$ with a function $k_{i j}(\boldsymbol{\theta})=k\left(t_{i}, t_{j}, \boldsymbol{\theta}\right)$ that parameterizes the covariance between values of the observable $\boldsymbol{y}(\boldsymbol{t})$ at the epochs $t_{i}$ and $t_{j}$ in $\boldsymbol{t}$. The observable $\boldsymbol{y}(\boldsymbol{t})$ has associated uncertainties $\boldsymbol{\sigma}(\boldsymbol{t})$ which were added along the diagonal of the covariance matrix $K$ in quadrature. The set of GP hyperparameters $\boldsymbol{\theta}$ are unique to the chosen covariance function $k_{i j}(\boldsymbol{\theta})$ and are solved for in the training step. After solving for the GP hyperparameters and thus obtaining a unique GP prior distribution, the GP prior conditioned on the data $y(t)$ becomes the predictive distribution. The mean function of the GP predictive distribution can be evaluated at previously unseen epochs $t^{*}$ using

$\boldsymbol{\mu}\left(\boldsymbol{t}^{*}\right)=K\left(\boldsymbol{t}^{*}, \boldsymbol{t}\right) \cdot K(\boldsymbol{t}, \boldsymbol{t})^{-1} \cdot \boldsymbol{y}(\boldsymbol{t})$,

which we took to be our GP activity model of the RVs by evaluating Eq. (1) at $\boldsymbol{t}$.

Because the stellar activity, and in particular the long-term photometric variation, is modulated by the stellar rotation period, we included a periodic term in our assumed covariance function $k_{i j}(\boldsymbol{\theta})$ with period equal to $P_{\text {rot }}$. We also included a radial component due to the stochastic temporal evolution of starspot lifetimes, spatial distributions, and contrasts thus forcing the covariance to not be strictly periodic. Explicitly the adopted covariance structure is parameterized by a quasi-periodic covariance kernel of the form

$k_{i, j}(\boldsymbol{\theta})=a^{2} \exp \left[-\frac{\left|t_{i}-t_{j}\right|^{2}}{2 \lambda^{2}}-\Gamma^{2} \sin ^{2}\left(\frac{\pi\left|t_{i}-t_{j}\right|}{P_{\mathrm{GP}}}\right)\right]$,

which is parameterized by four hyperparameters $\boldsymbol{\theta}=$ $\left(a, \lambda, \Gamma, P_{\mathrm{GP}}\right): a$ the amplitude of the correlations, $\lambda$ the exponential timescale, $\Gamma$ the coherence scale of the correlations, and $P_{\mathrm{GP}}$ the periodic timescale of the correlations which we interpret as $P_{\text {rot }}$. We also included an additional scalar jitter parameter $s$ which is added in quadrature to the diagonal elements of the covariance matrix $K$ such that $\theta$ becomes $\left(a, \lambda, \Gamma, P_{\mathrm{GP}}, s\right)$.

Using the Markov chain Monte-Carlo (MCMC) ensemble sampler emcee (Foreman-Mackey et al. 2013) we sample the marginalized posterior probability density functions (PDFs) of the five hyperparameters assuming uniform priors on the logarithm of each hyperparameter and maximizing the Gaussian logarithmic likelihood function

$\ln \mathcal{L}=-\frac{1}{2}\left(\boldsymbol{y}^{T} K^{-1} \boldsymbol{y}+\ln \operatorname{det} K+N \ln 2 \pi\right)$, 


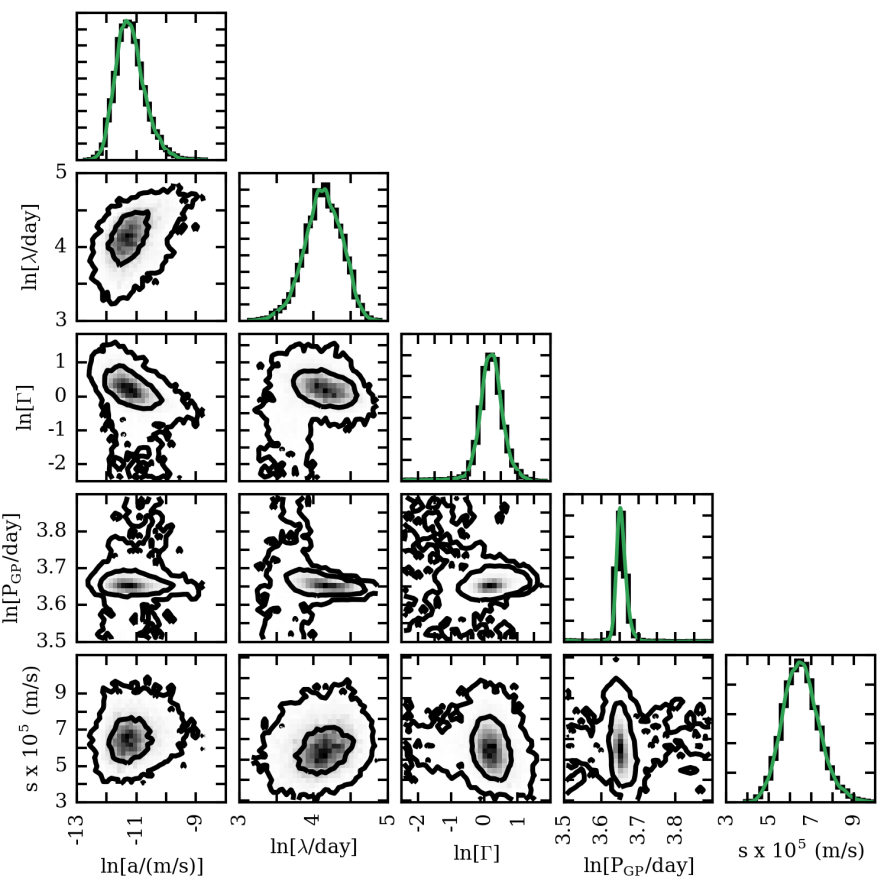

Fig. 3. Marginalized and joint posterior PDFs of the logarithmic GP hyperparameters used to model the K2 photometry shown in Fig. 1. Kernel density estimations of each model parameter's posterior are overlaid on the histograms with solid green lines.

where $\boldsymbol{y}$ is the vector of $N$ observations in the training set. In Model $1 \boldsymbol{y}=$ the binned photometric data points ${ }^{3}$ whereas $\boldsymbol{y}=$ BIS time series in Model 2.

The MCMC was initialized with 200 walkers and hyperparameter values $\left(a, \lambda, \Gamma, P_{\mathrm{GP}}\right)=(\max (\boldsymbol{y}-\langle\boldsymbol{y}\rangle)$, $10^{2}$ days, 1,36 days). We sampled the logarithmic hyperparameters up to $\approx 10$ autocorrelation times to ensure adequate convergence of the chains. We also monitored the acceptance fraction for each walker and insist that it lies within 20-50\%. The sampling of each hyperparameter's marginalized posterior PDF commences following a burn-in phase of the same duration. The resulting marginalized and joint posterior PDFs are shown in Fig. 3 along with kernel density estimations of each marginalized $1 D$ distribution. From the posterior PDF of $P_{\mathrm{GP}}$ we measured a stellar rotation period of $P_{\text {rot }}=38.6_{-0.4}^{+0.6}$ days.

\subsection{Joint modelling of RVs}

We proceeded with modelling the RVs jointly with Keplerian solutions for both K2-18b and c plus a trained quasi-periodic GP to model the correlated RV residuals attributed to stellar activity. The marginalized posterior PDFs of the GP hyperparameters $\lambda, \Gamma$, and $P_{\mathrm{GP}}$ from training were then used as informative priors in the joint RV analysis which treat the remaining GP hyperparameters $a$ and $s$ as free parameters. We sampled the informative priors using the kernel density estimations of each hyperparameter's PDF obtained during training. This methodology allows the model to learn the covariance structure of the stellar activity through observations which are independent of planetary sources and then apply that knowledge to the joint modelling of the RVs

\footnotetext{
Binning the $\mathrm{K} 2$ photometry in one day bins results in $N=78 \mathrm{com}-$ pared to the 3439 unbinned photometric observations thus drastically increasing the computational efficiency of the evaluating Eq. (3) in each step of the Markov chains.
}

thus distinguishing between stellar activity and planet-induced Doppler shifts.

The RV modelling is again performed using emcee. In Models 1, 2, and 3 our RV model consisted of 16 parameters including the five GP hyperparameters discussed in Sect. 4.1, the systemic velocity of K2-18 $\gamma_{0}$, the orbital periods of the two planets $P$, their times of inferior conjunction $T_{0}$, their RV semiamplitudes $K$, and the MCMC jump parameters $h=\sqrt{e} \cos \omega$ and $k=\sqrt{e} \sin \omega$ describing each planet's orbital eccentricity $e$ and argument of periastron $\omega$. This parameterization was chosen to minimize the correlation between $e$ and $\omega$ as well as reduce the tendency for the MCMC sampler to favour high-eccentricity solutions (Ford 2006). In Model 4 we only considered 11 model parameters as no GP activity model was included. Table 1 summarizes the adopted priors on each RV model parameter in each of the models considered in this study. We adopted non-informative priors for all Keplerian parameters other than the orbital period and time of mid-transit of K2-18b which were well-constrained by the transit light curve modelling in B17.

\section{Results}

\subsection{Results from RV data analysis}

Here we compare results from the four considered RV models. Each model contains Keplerian solutions for each of the two planets. Additionally Model 1 models the RV residuals with a quasi-periodic GP regression model that is trained on the K2 photometry in which the stellar rotation period is clearly detected (see Sect. 4.1). In this model the stellar rotation period, and hence the GP periodic term, is sufficiently distinct from the orbital period of K2-18b such that the two signals are not confused in our joint modelling and the measured semi-amplitude of K2-18b is not mis-estimated. Model 2 models the RV residuals with a quasi-periodic GP regression model that is trained on the BIS time series which is contemporaneous with the RVs. Model 3 models the RV residuals with an effectively unconstrained quasi-periodic GP and Model 4 neglects any modelling of the RV residuals therefore assuming that they are uncorrelated.

The maximum a posteriori (MAP) values of each model parameter along with the 16th and 84th percentiles of the marginalized posterior PDFs are reported in Table A.1. The marginalized and joint posterior PDFs of the model parameters used in Model 1 are shown in Fig. 4. In Sect. 5.2 we shall see that Model 1 is the best predictor of the observed RVs and therefore we only show the results from Model 1 in Fig. 4.

We emphasize that all Keplerian model parameters for the two planets are consistent at $1 \sigma$ across all four models. Recall that the stellar rotation period is only well-constrained in Model 1 via the K2 photometry and yet the measured semiamplitudes of $\mathrm{K} 2-18 \mathrm{~b}$ are consistent in each of the four models. This further demonstrates that there is minimal confusion between the RV signals at the stellar rotation period (38.6 days) and at the orbital period of K2-18b ( 32.93 days) both of which are not distinctly detected in the periodogram of the raw RVs (see top panel of Fig. 2) but appear to be hidden within a forest of peaks spanning periodicities between $\sim 25-45$ days. The consistency of all measured Keplerian parameters in each model also suggests that the RV residuals, following the removal of the two MAP Keplerian solutions, are weakly correlated because nearly identical RV solutions are obtained with and without a GP treatment of the RV residuals following the removal of our planet models. That is that $\mathrm{K} 2-18$ appears to be a spectroscopically 


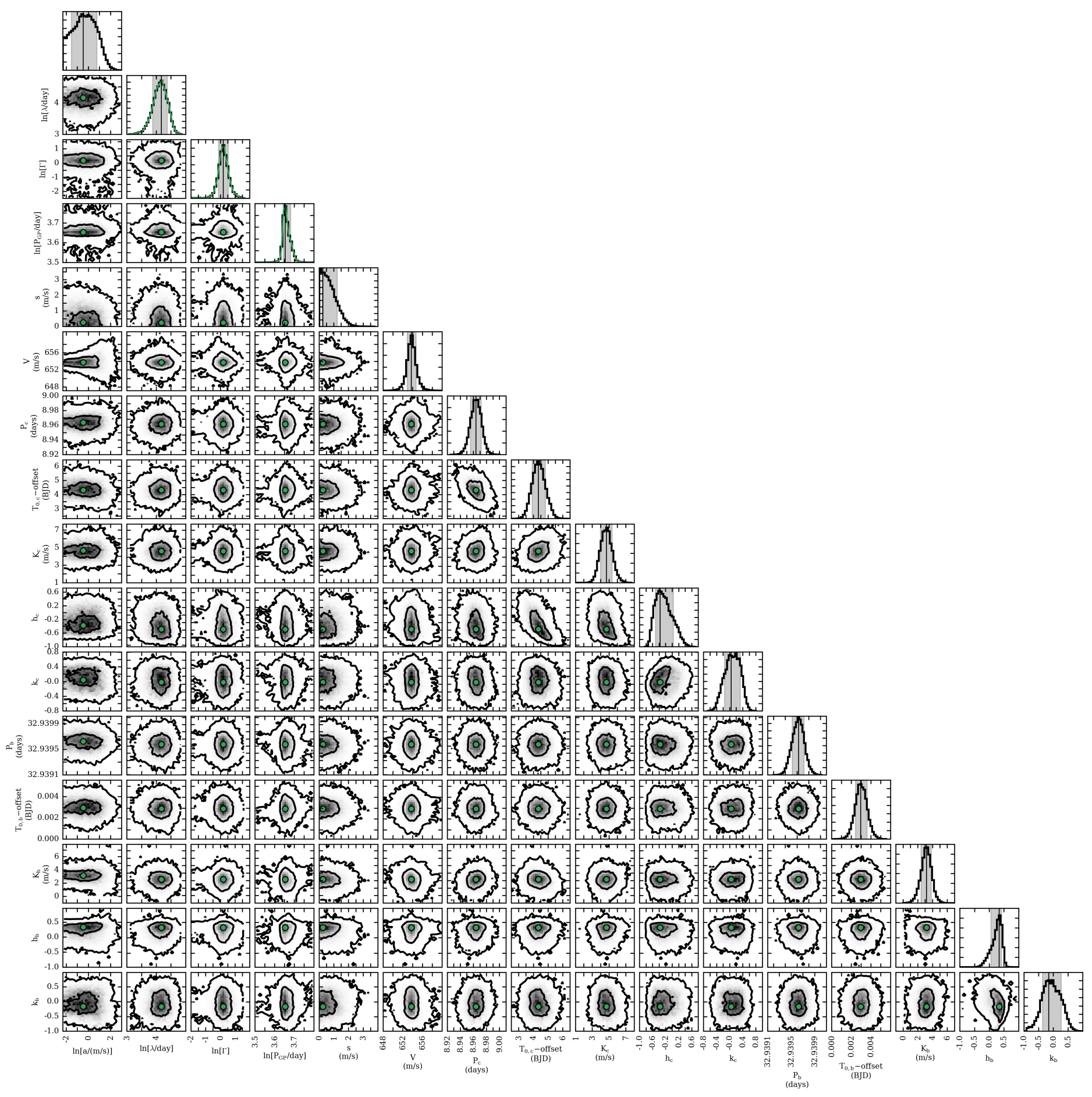

Fig. 4. The marginalized and joint posterior PDFs of the model parameters from Model 1 of the observed RVs. Model 1 of the observed RVs models the two planets with Keplerian orbital solutions and the residual RV activity signal with a GP regression model trained on the K2 photometry in Fig. 1. Kernel density estimations of the trained posteriors are shown in the histograms of the logarithmic GP hyperparameters $\lambda, \Gamma$, and $P_{\mathrm{GP}}$ (Cols. 2-4).

quiet star with the majority of its observed RV variation being attributable to planetary companions. Being spectroscopically quiet is promising for the prospect of transmission spectroscopy of $\mathrm{K} 2-18 \mathrm{~b}$; an observation that is significantly complicated by the presence of stellar activity. The quiet nature of K2-18 is highlighted by its low measured value of $\log R_{\mathrm{HK}}^{\prime}=-5.247$.

\subsection{RV model comparison}

A formal model comparison between the four considered models was performed using time-series cross-validation to compute the likelihood of each model given various training and testing subsets of the observed RVs (Arlot \& Celisse 2010). We split the RVs into chronological training sets with sizes ranging from 
Table 1. Summary of RV models and adopted priors.

\begin{tabular}{|c|c|}
\hline Parameter & Prior \\
\hline (2 planets + GP traine & = K2 photometry) \\
\hline $\begin{array}{l}\text { GP hyperparameters } \\
\text { Covariance amplitude, } a\left[\mathrm{~m} \mathrm{~s}^{-1}\right] \\
\text { Exponential timescale, } \lambda \text { [days] } \\
\text { Coherence, } \Gamma \\
\text { Periodic timescale, } P_{\mathrm{GP}} \text { [days] } \\
\text { Additive jitter, } s\left[\mathrm{~m} \mathrm{~s}^{-1}\right]\end{array}$ & $\begin{array}{c}\mathcal{J}(0.1,30)^{\bullet} \\
p(\lambda \mid \boldsymbol{y}) \\
p(\Gamma \mid \boldsymbol{y}) \\
p\left(P_{\mathrm{GP}} \mid \boldsymbol{y}\right) \\
\mathcal{J}\left(10^{-2}, 10\right)\end{array}$ \\
\hline $\begin{array}{l}\text { Keplerian parameters } \\
\gamma_{0}\left[\mathrm{~m} \mathrm{~s}^{-1}\right] \\
P_{\mathrm{b}}[\text { days }] \\
T_{0, \mathrm{~b}}[\mathrm{BJD}-2450000] \\
K_{\mathrm{b}}\left[\mathrm{m} \mathrm{s}^{-1}\right] \\
h_{\mathrm{b}}=\sqrt{e_{\mathrm{b}}} \cos \omega_{\mathrm{b}} \\
k_{\mathrm{b}}=\sqrt{e_{\mathrm{b}}} \sin \omega_{\mathrm{b}} \\
P_{\mathrm{c}}[\text { days }] \\
T_{0, \mathrm{c}}[\mathrm{BJD}-2450000] \\
K_{\mathrm{c}}\left[\mathrm{m} \mathrm{s}^{-1}\right] \\
h_{\mathrm{c}}=\sqrt{e_{\mathrm{c}}} \cos \omega_{\mathrm{c}} \\
k_{\mathrm{c}}=\sqrt{e_{\mathrm{c}}} \sin \omega_{\mathrm{c}}\end{array}$ & $\begin{array}{c}\mathcal{U}(620,670) \\
\mathcal{N}\left(32.939614,10^{-4}\right)^{\circ} \\
\mathcal{N}\left(7264.39144,6.3 \times 10^{-4}\right)^{\circ} \\
\bmod \mathcal{J}(1,20)^{*} \\
\mathcal{U}(-1,1)^{\dagger} \\
\mathcal{U}(-1,1)^{\dagger} \\
\mathcal{U}(8,10) \\
\mathcal{U}(7259,7269) \\
\bmod \mathcal{J}(1,20) \\
\mathcal{U}(-1,1)^{\dagger} \\
\mathcal{U}(-1,1)^{\dagger}\end{array}$ \\
\hline
\end{tabular}

Model 2

( 2 planets + GP trained on $\boldsymbol{y}=$ BIS)

see Model 1

Model 3

(2 planets + untrained GP)

see Model 1 with the

following modifications:

$\ln$ Exponential timescale, $\ln \lambda$ [days]

$\ln$ Coherence, $\ln \Gamma$

$\mathcal{U}(-10,10)$

$\ln$ Periodic timescale, $\ln P_{\mathrm{GP}}$ [days]

$\mathcal{U}(-3,3)$

$\mathcal{U}(3.2,4)$

\section{Model 4 \\ (2 planets)}

see Keplerian parameters in Model 1

Notes. ${ }^{\bullet} \mathcal{J}$ refers to a non-informative Jeffreys prior which is scale invariant; equal probability per decade which is necessary to sample multiple orders of magnitude (Gregory 2005). ${ }^{(\circ)}$ based on the transit light curve measurements from B17. ${ }^{(*)} \bmod \mathcal{J}(k, l) \mathrm{m} \mathrm{s}^{-1}$ refers to a modified Jefferys prior on a parameter $A$ which behaves like a uniform prior for $A \ll$ the knee at $\mathrm{k} \mathrm{m} \mathrm{s}^{-1}$ and behaves like a Jeffreys prior at $A \gg k$ up to $l$. We use a modified Jeffreys prior on the RV semi-amplitudes $K$ to sample multiple decades as a Jeffreys prior but also include $K=0 \mathrm{~m} \mathrm{~s}^{-1}$ which a Jeffreys prior does not (Gregory 2005). ${ }^{(\dagger)}$ We further insist that $e=h^{2}+k^{2}<1$.

20 measurements to the size of the full dataset less one (i.e. 74 measurements). The model parameters for each of the four considered models were optimized on the training set and the likelihood of the corresponding model is evaluated on the testing set. The testing set was simply the next observation chronologically following the final observation in the training set. The resulting median likelihood and median absolute deviation for each model is reported at the bottom of Table A.1 and was used to distinguish which of our four RV models performs optimally on the prediction of unseen RV measurements and thus best fits the data without over-fitting. Through time-series crossvalidation we find that Model 1 is the best predictor of the observed RVs. In the remainder of this study we consider the results from Model 1 to be the measured values of the planets K2-18b and c.

To confirm that we have detected a second planet K2-18c in our RV data, we performed a second round of time-series cross-validation calculations. In these calculations we compared three RV models each containing 0,1 , or 2 planets. We considered $\mathrm{K} 2-18 \mathrm{~b}$ to be the lone planet in the one planet model. In each model we also considered a GP activity model that was trained on the K2 photometry as was Model 1 above. Following the same methodology as previously discussed we find median logarithmic likelihoods of $\ln \mathcal{L}_{0}=-2.693 \pm 0.056, \ln \mathcal{L}_{1}=$ $-2.642 \pm 0.047$, and $\ln \mathcal{L}_{2}=-2.566 \pm 0.026$. From this we find that $\ln \mathcal{L}_{2}-\ln \mathcal{L}_{1}=0.076 \pm 0.054>0$ therefore arguing that the two planet model is the best predictor of unseen RV measurements and confirming that our two planet model containing both $\mathrm{K} 2-18 \mathrm{~b}$ and $\mathrm{c}$ is the RV model most favoured by the data.

The contributions to the observed RVs from stellar activity and each planet were depicted in Fig. 5. Together these physical models account for all significant periodicities in the observed RVs. In Fig. 5 we show the raw RVs as well as the RVs corrected for the individual RV component and compare each time series to its MAP model. In panel $\mathrm{b}$ we can see the relative importance of the GP activity model at modelling the RV residuals following the removal of our two planet model. Owing to the spectroscopically quiet nature of K2-18, the contribution to the observed RVs from activity is relatively small yet still holds a periodic manifestation at the stellar rotation period, albeit with a small amplitude. The residual rms following the removal of all modelled contributions is $2.89 \mathrm{~m} \mathrm{~s}^{-1}$. This value is less than the median photon noise limit of the measured RVs of $3.56 \mathrm{~m} \mathrm{~s}^{-1}$ suggesting that we have modelled all significant RV contributions. For comparison, the residual rms achieved in Model 4, which neglects any red noise modelling following the removal of the two Keplerian solutions, is $3.16 \mathrm{~m} \mathrm{~s}^{-1}$. This value is also less than the median photon noise limit suggesting that the GP regression modelling alone in Models 1, 2, and 3 does not result in over-fitting of the data.

The Model 1 MAP Keplerian orbital solutions for K2-18b and c, but with eccentricities fixed to zero, are shown in Fig. 6. Here we report circular orbital solutions given that with these data we can only place upper limits on each planet's eccentricity rather than detect it directly. The RV data are phase-folded to each planet's MAP orbital period and time of inferior conjunction and are corrected for stellar activity using the mean GP activity model trained on the K2 photometry and assuming a mean model equal to the superposition of the two MAP Keplerian solutions.

\section{Searching for transits of K2-18c}

From our RV analysis in Sect. 5 we derived the approximate linear ephemeris of K2-18c. We can therefore predict the passage of K2-18c at inferior conjunctions within the mostly continuous K2 photometric monitoring shown in Fig. 1. In Fig. 1 we indicate the nine such passages of K2-18c. Given the comparable minimum masses of $\mathrm{K} 2-18 \mathrm{~b}$ and $\mathrm{c}$, it is reasonable to expect that the two planets also have comparable radii (recall $r_{\mathrm{p}, \mathrm{b}} \sim 2.38 R_{\oplus}$ ). Furthermore (Ciardi et al. 2013) argued that Kepler multi-planet systems with planet radii $\lesssim 3 R_{\oplus}$ do not exhibit a size - semimajor axis correlation such that the inner K2-18c is not expected to have undergone significant atmospheric escape compared to $\mathrm{K} 2-18 \mathrm{~b}$. However the two $10 \sigma$ transits of $\mathrm{K} 2-18 \mathrm{~b}$ are clearly discernible by-eye in the K2 photometry whereas the predicted transits of K2-18c are not. This suggests that either K2-18c is 


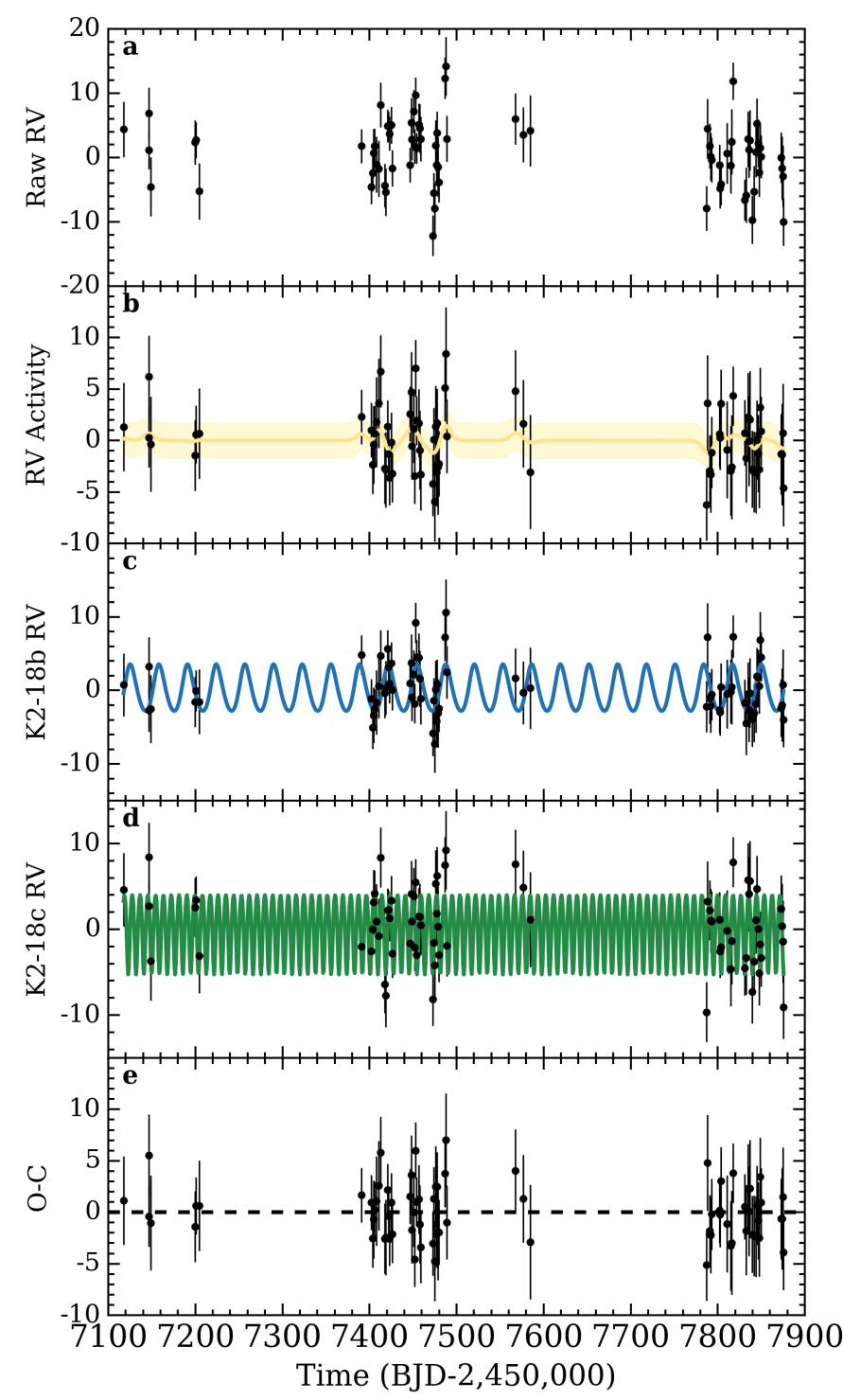

Fig. 5. Panel $a$ : Raw RVs less the systemic velocity of K2-18. Panel $b$ : RV contribution from stellar activity; raw RVs corrected by the MAP Keplerian orbital solutions for each detected planet. Panel $c$ : RV contribution from K2-18b; raw RVs corrected for activity and K2-18c. Panel d: RV contribution from $\mathrm{K} 2-18 \mathrm{c}$; raw RVs corrected for activity and K2-18b. Panel $e$ : the RV residuals. The solid curves in panels $b$, $c$, and $d$ depict the mean GP activity model, and the MAP Keplerian models for K2-18b and $c$ ) respectively. The surrounding shaded region in panel $b$ is the $68 \%$ confidence interval on the mean GP model. All $\mathrm{RV}$ units are in $\mathrm{ms}^{-1}$.

much smaller than $\mathrm{K} 2-18 \mathrm{~b}$ such that its resulting transit depth is below the threshold for detection, or that the orbit K2-18c is mutually inclined with that of $\mathrm{K} 2-18 \mathrm{~b}$ such that it misses a transit configuration.

Here we attempt to confirm that $\mathrm{K} 2-18 \mathrm{c}$ is indeed not transiting in the K2 light curves. To do so we perform an MCMC sampling of the K2-18c transit model parameters $\left(P_{\mathrm{c}}, T_{0, \mathrm{c}}\right.$, $r_{\mathrm{p}, \mathrm{c}} / R_{\mathrm{s}}, a_{\mathrm{c}} / R_{\mathrm{s}}$, and impact parameter $b_{\mathrm{c}}$ ) using the $\mathrm{K} 2$ photometry and following the removal of the known transits of K2-18b. A quadratic limb-darkening law is assumed with fixed parameters in the Kepler bandpass: $a=0.3695$ and $b=0.3570$. These values are interpolated from the tables of Claret \& Bloemen (2011)

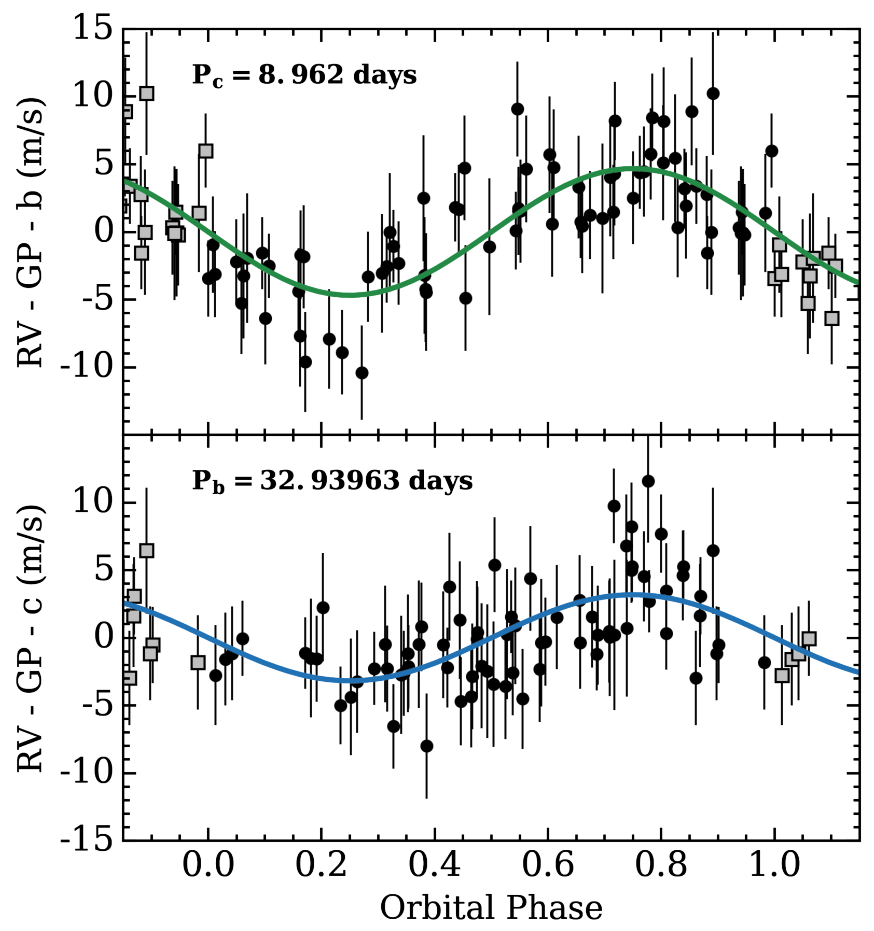

Fig. 6. Phase-folded RVs for each planet in the K2-18 planetary system (top: K2-18c and bottom: K2-18b). The RVs have been corrected for stellar activity with a quasi-periodic GP model trained on the K2 photometry. The [solid_curves, depict the maximum a-posteriori Keplerian orbital solutions for each planet with fixed circular orbits.

based on the known K2-18 surface gravity and effective temperature. In each MCMC step we compute the corresponding transit model using the batman implementation (Kreidberg 2015) of the Mandel \& Agol (2002) transit model. We assumed a circular orbit of K2-18c and adopt the same MCMC methodology utilized on the RV data in Sect. 4.2. The orbital period and time of inferior conjunction (i.e. time of mid-transit) were sampled from their joint RV posterior which maintains their apparent correlation (see Fig. 4). Priors on the scaled planet radius, and impact parameter are assumed uniform. In this way the scaled planetary radius is uncorrelated with its measured minimum mass and the impact parameter is constrained to be $\left|b_{\mathrm{c}}\right|<1$ as is required for a transit to occur.

Based on our MCMC analysis we find that the values of $r_{\mathrm{p}, \mathrm{c}} / R_{\mathrm{s}}$ are consistent with zero, that is, no transit is detected in the K2 data. Assuming the most likely value of $R_{\mathrm{s}}=0.411 R_{\odot}$, we calculate a planet radius upper limit of $r_{\mathrm{p}, \mathrm{c}}<0.52 R_{\oplus}$ at $99 \%$ confidence assuming that $\mathrm{K} 2-18 \mathrm{c}$ is indeed transiting. However, if K2-18c were this size and transiting, albeit unknowingly due to its small size, the planet would have a bulk density of $\gtrsim 295 \mathrm{~g} \mathrm{~cm}^{-3}$ or $\gtrsim 54 \rho_{\oplus}$; an unphysically large value given the compressibility of pure iron. Thus we conclude that K2-18c is not transiting in the $\mathrm{K} 2$ data and is therefore non-coplanar with K2-18b despite having a smaller orbital separation.

To visualize the data a selection of light curve models are compared to the phase-folded K2 photometry in Fig. 7. Models shown include a suite of $r_{\mathrm{p}, \mathrm{c}}$ values including its upper limit derived from MCMC $\left(0.52 R_{\oplus}\right), 0.75 R_{\oplus}, 1 R_{\oplus}$, and the radius of $\mathrm{K} 2-18 \mathrm{~b}\left(2.38 R_{\oplus}\right)$ which is detected at the $10 \sigma$ level in the $\mathrm{K} 2$ photometry (Montet et al. 2015). 


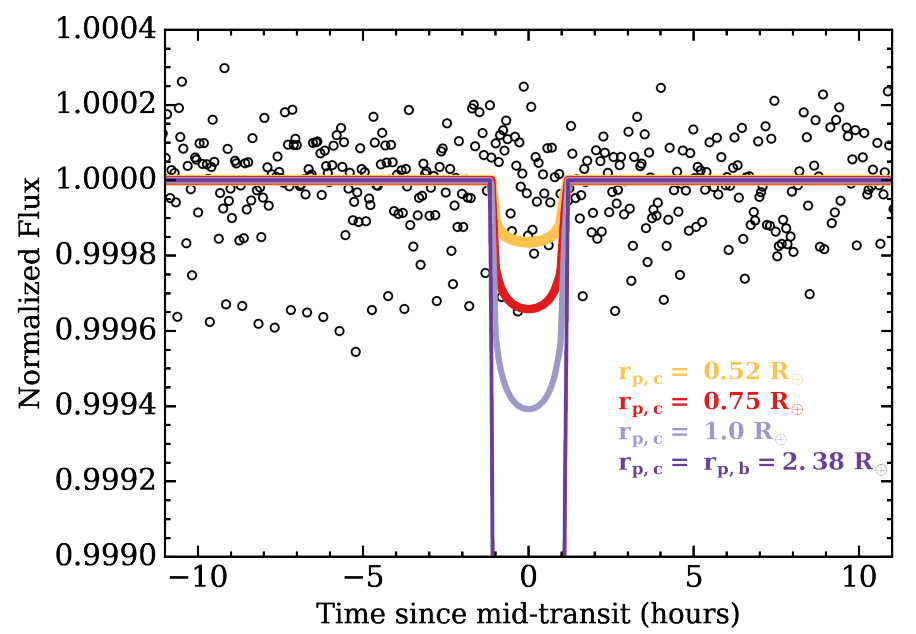

Fig. 7. K2 light curve of K2-18 phase-folded to the maximum aposteriori orbital period and time of mid-transit of $\mathrm{K} 2-18 \mathrm{c}$ from Model 1. Four transit light curve models are over-plotted for various illustrative values of the assumed size of K2-18c: the $99 \%$ upper limit

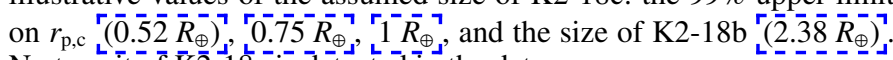
No transit of $\overline{\mathrm{K}} \overline{2}-1+\overline{\mathrm{c}}$ is $\overline{\text { detected }}$ in the data.

\section{Dynamical stability and eccentricity restrictions}

The non-detection of K2-18c in transit (see Sect. 6) suggests that its orbital plane is not perfectly coplanar with the outer transiting $\mathrm{K} 2-18 \mathrm{~b}$ whose semi-major axis is $\sim 2.4$ times greater than $\mathrm{K} 2-18 \mathrm{c}$ 's. The orbital inclination of K2-18b is $89.5785_{-0.0088}^{+0.0079} \mathrm{deg}$ with a corresponding impact parameter of $0.601_{-0.011}^{+0.013}$ (B17). In order for the orbit of K2-18c to not pass in front of its host star its orbital inclination must be tilted either $\gtrsim 1.41^{\circ}$ or $\lesssim-2.25^{\circ}$ from the orbit of K2-18b depending on which hemisphere of the stellar disk its transit chord will traverse. Such a mutual inclination is consistent with the peak in the distribution of Kepler multi-planet mutual inclinations (Figueira et al. 2012; Fabrycky et al. 2014). If indeed the planetary angular momentum vectors are within only a few degrees and therefore nearly aligned then we can analytically evaluate their Hill stability given estimates of their orbital eccentricities and assuming an inclination correction factor that is close to unity (Gladman 1993). If we assume the simplest case of initially circular orbits then the system is strongly Hill stable given that the two planets are currently separated by $\sim 23$ mutual Hill radii.

Accurate orbital eccentricities of small planets with precision radial velocities are notoriously difficult to measure. For example, the change in RV semi-amplitude of a circular K2-18b compared to an eccentricity of 0.1 is $\lesssim 2 \mathrm{~cm} \mathrm{~s}^{-1}\left(\sim 0.5 \%\right.$ of $\left.K_{\mathrm{b}}\right)$ or $15 \mathrm{~cm} \mathrm{~s}^{-1}\left(\sim 5 \%\right.$ of $\left.K_{\mathrm{b}}\right)$ for an eccentricity of 0.3 . The aforementioned values are both at least an order of magnitude smaller than the characteristic RV uncertainty of the HARPS measurements presented in this work. Given that the system is Hill stable at small eccentricities we can use dynamical simulations to constrain the orbital eccentricities of the planets insisting that the system remain stable throughout its simulated evolution.

To constrain the planet eccentricities we performed a suite of $10^{4}$ dynamical integrations wherein we sample linearly each planet's $e \in[0,1)$. In each simulation the orbital inclination of $\mathrm{K} 2-18 \mathrm{~b}$ is drawn from $\mathcal{N}\left(89.5785^{\circ}, 0.0084^{\circ}\right)$ while the system's mutual inclination is drawn from $\mathcal{N}\left(\Delta i_{\text {min,c }}, 1.5^{\circ}\right)$ such that the planet inclinations remain uncorrelated with the orbital eccentricities thus permitting an unbiased assessment of the system's stability across the Keplerian parameter space. We insist

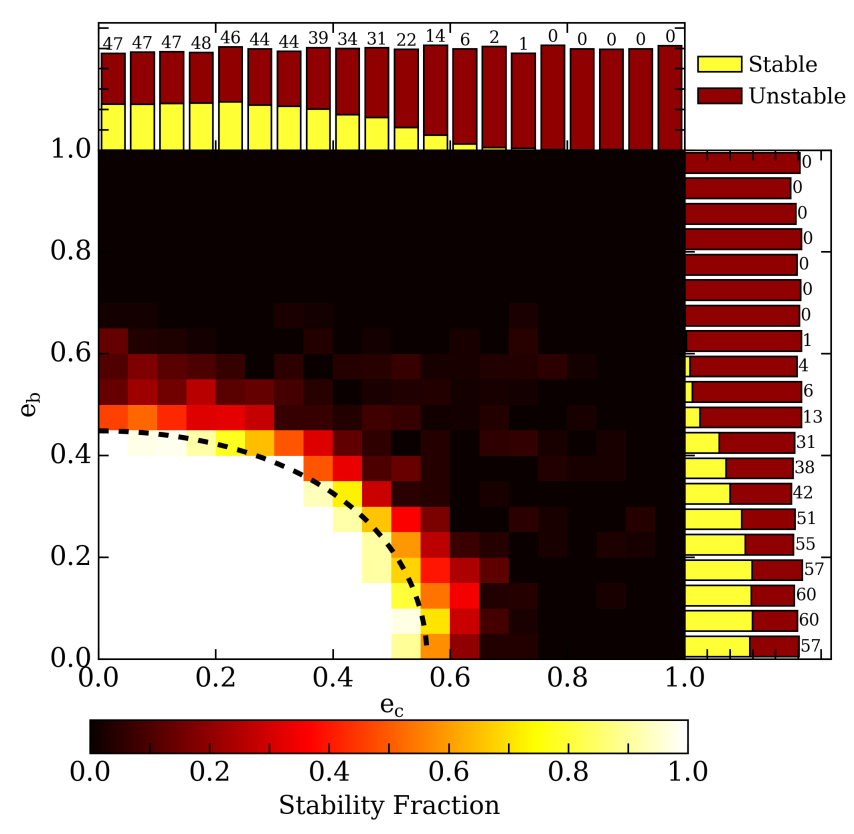

Fig. 8. Smoothed 2D map depicting the fraction of stable systems as a function of each planet's eccentricity based on a suite of dynamical integrations. The dashed black curve depicts the analytic condition for Hill stability from Gladman (1993) assuming the MAP masses and semimajor axes from Model 1 in Table A.1. 1D histograms depict the number of stable and unstable (yellow and red respectively) systems in eccentricity bins of width 0.05 and marginalized over all other dynamical parameters. The annotated numbers report each bin's stability fraction in percentages.

that $\mathrm{K} 2-18 \mathrm{c}$ be non-transiting at the start of each simulation by setting $\Delta i_{\text {min,c }}$ to be the minimum mutual inclination required for $\left|b_{\mathrm{c}}\right|>1$ and rejecting draws for which this is not true. The dispersion in sampled mutual inclinations is tuned such that the mode of the resulting distribution lies within $\sim 1-2^{\circ}$ (Fabrycky et al. 2014). Each planet's initial semi-major axis, true anomaly, and absolute mass is drawn from a Gaussian distribution with a mean value equal to the parameter's MAP value from Model 1 in Table A.1 and with standard deviations equal to its average measurement uncertainty. The stellar mass is drawn from $\mathcal{N}(0.359,0.047) M_{\odot}$. The ascending node longitudes and arguments of periapsis are both drawn from $\mathcal{U}(-\pi, \pi)$. The system is then integrated forward in time from the epoch of the first $\mathrm{K} 2$ photometric observation $\left(\mathrm{BJD}=2456810.26222\right.$ ) for $10^{6} \mathrm{yr}$ using the Wisdom-Holman symplectic integrator WHFast (Rein \& Tamayo 2015) implemented in the open-source REBOUND Nbody package (Rein \& Liu 2012). These integrations are not intended to provide a comprehensive overview of the system's dynamical stability but rather are useful to show that the system can remain stable up to at least $1 \mathrm{Myr}$ and provide constraints on the planet eccentricities.

We classify stable integrations as those in which the minimum distance between the planets never becomes less than their mutual Hill radius. The fraction of stable systems as a function of each planet's eccentricity and marginalized over all other dynamical parameters is shown in Fig. 8. Strong correlations between the fraction of stable systems and dynamical parameters other than planet eccentricities was not apparent so we focus here on the effect of eccentricities only. At small eccentricities there is a large stable region wherein the fraction of systems that remain stable is $\gtrsim 80 \%$ and the system is known to be Hill stable 


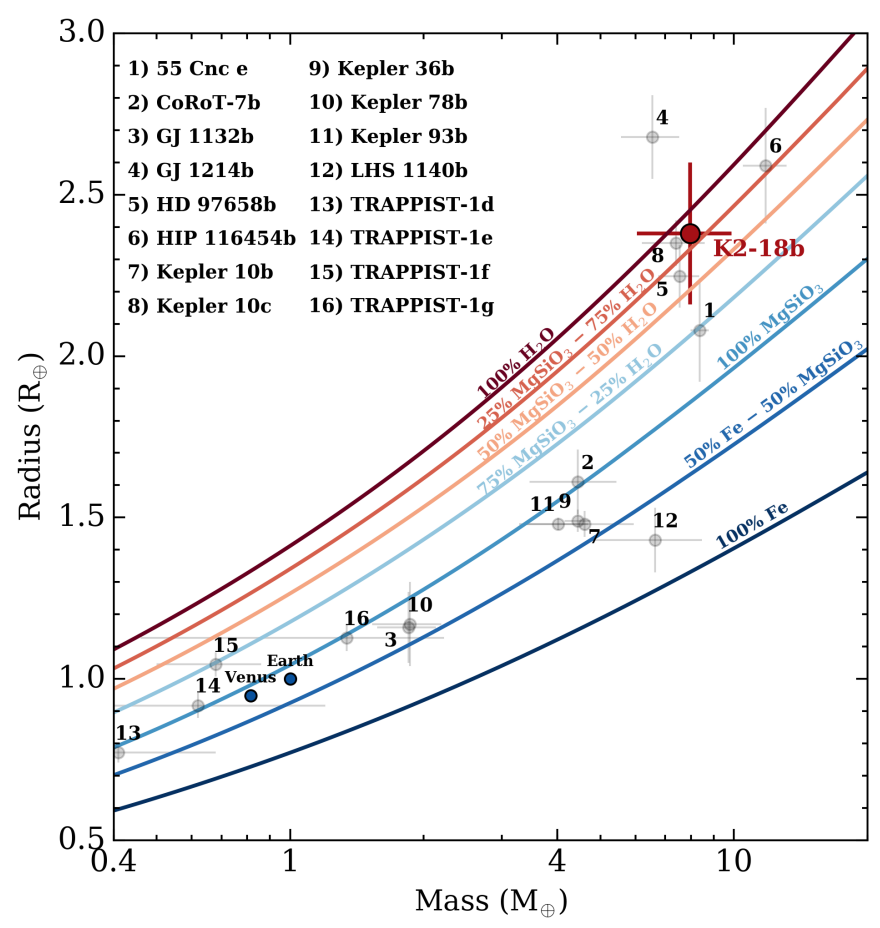

Fig. 9. K2-18b along with a sample of other small exoplanets in the planetary mass and radius space. Overlaid curves are two-component interior structure models of fully-differentiated solid planets with mass fractions annotated for each curve.

based on the analytic criterion. As we increase either planet's eccentricity the fraction of stable systems decreases. This is also illustrated by further marginalizing over planet eccentricities and considering the one-dimensional representations of each system's stability fraction in the histograms shown in Fig. 8.

The RV analysis discussed Sect. 5 and our dynamical simulations provide two independent methods for constraining the orbital eccentricities of the K2-18 planets. We can therefore combine these independent results by using the dynamical stability fractions shown in Fig. 8 as an additional prior on the $i$ th planet's derived eccentricity posterior: $e_{i}=h_{i}^{2}+k_{i}^{2}$. To do this we resample each planet's RV eccentricity posterior and accept draws with a probability equal to the stability fraction at that drawn eccentricity value \pm 0.025 . This choice of bin width was varied between 0.01 and 0.1 and was found not to have a significant effect on the results. In this way numerous random samples from each planet's RV eccentricity posterior are rejected due to the low corresponding stability fraction. This is especially true for large eccentricities wherein the system no longer satisfies the Hill stability criterion. From the modified eccentricity posteriors we can calculate the 99th percentiles and find that $e_{\mathrm{b}}<0.43$ and $e_{\mathrm{c}}<0.47$ at that confidence level. These are the eccentricity values reported in Table A.1 and represent a more stringent evaluation of each planet's eccentricity than considering the RV data alone.

\section{Discussion}

With a set of 75 precision radial velocity measurements taken with the HARPS spectrograph we have obtained a robust mass measurement of the transiting HZ super-Earth K2-18b and detected a second super-Earth K2-18c. The orbit of the newly discovered K2-18c lies interior to that of K2-18b and yet the planet is non-transiting. This implies that the orbital planes of the planets are mutually inclined. In order for K2-18c to not be seen in-transit the planetary system requires a mutual inclination of just $\gtrsim 1.4^{\circ}$ which is consistent with the observed distribution of mutually inclined multi-planet systems (Figueira et al. 2012; Fabrycky et al. 2014). Dynamical simulations of the system revealed that the oscillation timescale of the planets' orbital inclinations is $O\left(10^{6} \mathrm{yr}\right)$ suggesting that it may take many years before $\mathrm{K} 2-18 \mathrm{c}$ reaches a transiting configuration. Although exactly how long depends sensitivity on its current inclination which remains unknown. The discovery of RV planets in transiting M dwarf planetary systems further emphasizes the prevalence of multiple Earth to super-Earth-sized planets around nearby $\mathrm{M}$ dwarfs and that these additional planets can be uncovered with moderate RV follow-up (Cloutier et al. 2017). Multi-planet systems such as K2-18 provide unique opportunities to study planet formation processes around $\mathrm{M}$ dwarfs via direct comparative planetology.

The presence of a second planet in the K2-18 transiting system will result in mutual planetary interactions thus making the orbit of K2-18b non-Keplerian and possibly resulting in an observable transit timing variation (TTV). Assuming a mutual inclination of $\mathrm{K} 2-18 \mathrm{c}$ that just misses a transiting configuration, we estimate the expected TTVs of K2-18b using the TTVFaster package (Deck et al. 2014; Agol \& Deck 2016). We adopted the maximum a-posteriori masses and orbital periods of the two planets from Model 1 and uniformly sample their eccentricities up the $99 \%$ upper limits reported in Table A.1. The remaining orbital parameters of the planets that are unconstrained by the RV data are sampled uniformly between 0 and $2 \pi$. We find a maximum TTV for K2-18b of $\sim 40 \mathrm{~s}$ which is slightly less than, but of the same order as the uncertainty in its measured time of mid-transit ( $\sim 50 \mathrm{~s}$; B17). Thus with photometric monitoring of at least comparable quality to the K2 photometry shown in Fig. 1, detecting TTVs in the K2-18 multi-planet system is unlikely to provide any significant new insight into the nature of the system. Indeed no significant TTVs were observed in the Spitzer light curves from B17.

With our measured mass of K2-18b the planet joins a select group of $\mathrm{HZ}$ planets with constraints on both its mass and radius. This represents a significant step towards searching for potentially habitable planets around stars earlier than $\sim$ M4 (Dittmann et al. 2017). With its maximum a posteriori mass of $7.96 \pm 1.91 M_{\oplus}$ the bulk density of K2-18b $\left(\rho_{\mathrm{p}, \mathrm{b}}=\right.$ $3.3 \pm 1.2 \mathrm{~g} \mathrm{~cm}^{-3}$ ) lies between that of an Earth-like rocky planet and a low density Neptune-like planet. The planet is therefore likely too large to be a terrestrial Earth-like planet (Valencia et al. 2007; Fulton et al. 2017). Including K2-18b on the exoplanet mass-radius diagram in Fig. 9 we find that the internal structure of K2-18b is consistent with a range of two-component solidplanet models (Zeng \& Sasselov 2013) owing to the uncertainty in its measured radius and mass which is at the level of $\sim 24 \%$. In particular, the $1 \sigma$ lower mass limit of $\mathrm{K} 2-18 \mathrm{~b}$ permits it to be a complete "water world" even though its upper mass limit is consistent with a largely rocky interior surrounded by a significant mass fraction of water ice. In this parameter space the physical parameters of $\mathrm{K} 2-18 \mathrm{~b}$ are most similar to the superEarths HD 97658b (Van Grootel et al. 2014) and Kepler 10c (Dumusque et al. 2014; Rajpaul et al. 2017) despite receiving $\sim 65$ and $\sim 24$ times less insolation than those two planets respectively. Furthermore K2-18b is of a similar mass to the habitable zone planet LHS 1140b (Dittmann et al. 2017) and receives a comparable level of insolation despite being $\sim 1.6$ times larger than LHS 1140b. Analyzing the mass-radius relationship of these small planets over a range of equilibrium temperatures is a critical step towards understanding which of these systems 
have retained significant atmospheric content thus making them more suitable to extraterrestrial life.

Distinguishing between K2-18b as a pure water-world or a scaled-up Earth with a significant gaseous envelope will likely require transmission spectroscopy follow-up observations either with high-resolution spectrographs from the ground or from space with JWST. With $J=9.8, H=9.1$, and $K=8.9$ (Cutri et al. 2003), we stress that K2-18 is currently the second brightest $\mathrm{M}$ dwarf with a transiting habitable zone planet behind the recently discovered LHS $1140 \mathrm{~b}$. In the coming years the sample of habitable zone $\mathrm{M}$ dwarf planets is expected to increase dramatically following the launch of TESS (Ricker et al. 2014), although the majority of TESS planets will be more distant than LHS 1140 (Sullivan et al. 2015).

Considering the prospect of observational follow-up of $\mathrm{K} 2-18 \mathrm{~b}$ in transmission spectroscopy, if we consider an atmosphere that is cloud-free and dominated by hydrogen, then spectral features from well-mixed near-IR absorbing species such as water would have amplitudes of $\Delta F / F \sim 10 H r_{\mathrm{p}} / R_{\mathrm{s}}^{2} \sim 230 \mathrm{ppm}$ where $H=k_{\mathrm{B}} T_{\text {eq }} / \mu g$ is the atmospheric scale height, $T_{\text {eq }}$ is the planet's equilibrium temperature set by the stellar insolation, $\mu$ is the mean molecular weight of the atmosphere, and $g$ is the surface gravity (Miller-Ricci et al. 2009; Kaltenegger \& Traub 2009). If instead the atmosphere is dominated by heavier elements, similar Earth's (e.g. $\mathrm{N}_{2}+\mathrm{O}_{2}, \mu=29$ ), then the transmission signal will be significantly smaller $(\sim 10 \mathrm{ppm})$ though potentially still detectable with JWST with several visits. Because of the brightness of its host star and the low bulk density of K2-18b, the system offers a unique opportunity to study super-Earth atmospheres receiving Earth-like insolation in the JWST-era.

Acknowledgements. R.C. is partially supported in this work by the National Science and Engineering Research Council of Canada and the Institute for Research on Exoplanets. R.C. would like to thank Dan Tamayo for his useful discussions regarding the dynamical simulations presented in this work and their interpretation. X.B., X.D., and T.F. acknowledge the support of CNRS/PNP (Programme national de planétologie). D.E. acknowledges support from the National Centre for Competence in Research "PlanetS" of the Swiss National Science Foundation (SNSF) and from the European Research Council (ERC) under the European Union's Horizon 2020 research and innovation programme (grant agreement No. 724427). X.B., J.M.A. and A.W. acknowledge funding from the European Research Council under the ERC Grant Agreement No. 337591-ExTrA. N.C.S. acknowledges support from Fundação para a Ciência e a Tecnologia (FCT) through national funds and from FEDER through COMPETE2020 by the following grants: UID/FIS/04434/2013 \& POCI-01-0145-FEDER-007672 and PTDC/FIS-AST/1526/2014 \& POCI-010145-FEDER-016886. NCS also acknowledges the support from FCT through Investigador FCT contract IF/00169/2012/CP0150/CT0002. Some of the data presented in this paper were obtained from the Mikulski Archive for Space Telescopes (MAST). STScI is operated by the Association of Universities for Research in Astronomy, Inc., under NASA contract NAS5-26555. Support for MAST for non-HST data is provided by the NASA Office of Space Science via grant NNX09AF08G and by other grants and contracts.

\section{References}

Agol, E., \& Deck, K. 2016, ApJ, 818, 177

Aigrain, S., Pont, F., \& Zucker, S. 2012, MNRAS, 419, 3147
Arlot, S., \& Celisse, A. 2010, Statist. Surv., 4, 40

Astudillo-Defru, N., Bonfils, X., Delfosse, X., et al. 2015, A\&A, 575, A119 Astudillo-Defru, N., Delfosse, X., Bonfils, X., et al. 2017, A\&A, 600, A13 Baliunas, S. L., Donahue, R. A., Soon, W. H., et al. 1995, ApJ, 438, 269 Baranne, A., Queloz, D., Mayor, M., et al. 1996, A\&AS, 119, 373 Beichman, C., Benneke, B., Knutson, H., et al. 2014, PASP, 126, 1134 Benneke, B., Werner, M., Petigura, E., et al. 2017, ApJ, 834, 187 Bonfils, X., Mayor, M., Delfosse, X., et al. 2007, A\&A, 474, 293 Bouchy, F., Pepe, F., \& Queloz, D. 2001, A\&A, 374, 733

Ciardi, D. R., Fabrycky, D. C., Ford, E. B., et al. 2013, ApJ, 763, 41

Claret, A., \& Bloemen, S. 2011, A\&A, 529, A75

Cloutier, R., Doyon, R., Menou, K., et al. 2017, AJ, 153, 9

Cutri, R. M., Skrutskie, M. F., van Dyk, S., et al. 2003, 2MASS All Sky Catalog of point sources

Deck, K. M., Agol, E., Holman, M. J., \& Nesvorný, D. 2014, ApJ, 787, 132

Dittmann, J. A., Irwin, J. M., Charbonneau, D., et al. 2017, Nature, 544, 333 Dressing, C. D., \& Charbonneau, D. 2015, ApJ, 807, 45

Dumusque, X., Bonomo, A. S., Haywood, R. D., et al. 2014, ApJ, 789, 154

Fabrycky, D. C., Lissauer, J. J., Ragozzine, D., et al. 2014, ApJ, 790, 146

Faria, J. P., Haywood, R. D., Brewer, B. J., et al. 2016, A\&A, 588, A31

Figueira, P., Marmier, M., Boué, G., et al. 2012, A\&A, 541, A139

Ford, E. B. 2006, ApJ, 642, 505

Foreman-Mackey, D., Hogg, D. W., Lang, D., \& Goodman, J. 2013, PASP, 125, 306

Foreman-Mackey, D., Montet, B. T., Hogg, D. W., et al. 2015, ApJ, 806, 215

Fulton, B. J., Petigura, E. A., Howard, A. W., et al. 2017, AJ, 154, 109

Gaidos, E., Mann, A. W., Kraus, A. L., \& Ireland, M. 2016, MNRAS, 457, 2877

Gladman, B. 1993, Icarus, 106, 247

Gregory, P. C. 2005, ApJ, 631, 1198

Henden, A., \& Munari, U. 2014, Contributions of the Astronomical Observatory Skalnate Pleso, 43, 518

Kaltenegger, L., \& Traub, W. A. 2009, ApJ, 698, 519

Kaltenegger, L., Segura, A., \& Mohanty, S. 2011, ApJ, 733, 35

Kasting, J. F., Whitmire, D. P., \& Reynolds, R. T. 1993, Icarus, 101, 108

Kitchatinov, L. L., \& Olemskoy, S. V. 2011, MNRAS, 411, 1059

Kopparapu, R. K., Ramirez, R., Kasting, J. F., et al. 2013, ApJ, 765, 131 Kreidberg, L. 2015, PASP, 127, 1161

Kreidberg, L., Bean, J. L., Désert, J.-M., et al. 2014, Nature, 505, 69

Lovis, C., \& Pepe, F. 2007, A\&A, 468, 1115

Luger, R., Agol, E., Kruse, E., et al. 2016, AJ, 152, 100

Mandel, K., \& Agol, E. 2002, ApJ, 580, L171

Mayor, M., Pepe, F., Queloz, D., et al. 2003, The Messenger, 114, 20

Miller-Ricci, E., Seager, S., \& Sasselov, D. 2009, ApJ, 690, 1056

Montet, B. T., Morton, T. D., Foreman-Mackey, D., et al. 2015, ApJ, 809, 25

Newton, E. R., Irwin, J., Charbonneau, D., et al. 2016, ApJ, 821, 93

Pepe, F., Mayor, M., Galland, F., et al. 2002, A\&A, 388, 632

Pepe, F., Mayor, M., Queloz, D., et al. 2004, A\&A, 423, 385

Rajpaul, V., Buchhave, L. A., \& Aigrain, S. 2017, MNRAS, 471, L125

Rein, H., \& Liu, S.-F. 2012, A\&A, 537, A128

Rein, H., \& Tamayo, D. 2015, MNRAS, 452, 376

Ricker, G. R., Winn, J. N., Vanderspek, R., et al. 2014, in Space Telescopes and Instrumentation 2014: Optical, Infrared, and Millimeter Wave, Proc. SPIE, 9143, 914320

Rodler, F., \& López-Morales, M. 2014, ApJ, 781, 54

Rogers, L. A. 2015, ApJ, 801, 41

Scargle, J. D. 1982, ApJ, 263, 835

Stevenson, K. B., Harrington, J., Nymeyer, S., et al. 2010, Nature, 464, 1161

Sullivan, P. W., Winn, J. N., Berta-Thompson, Z. K., et al. 2015, ApJ, 809, 77

Valencia, D., Sasselov, D. D., \& O'Connell, R. J. 2007, ApJ, 665, 1413

Valencia, D., Guillot, T., Parmentier, V., \& Freedman, R. S. 2013, ApJ, 775, 10

Van Grootel, V., Gillon, M., Valencia, D., et al. 2014, ApJ, 786, 2

Wilson, O. C. 1968, ApJ, 153, 221

Zacharias, N., Finch, C. T., Girard, T. M., et al. 2013, AJ, 145, 44

Zeng, L., \& Sasselov, D. 2013, PASP, 125, 227 


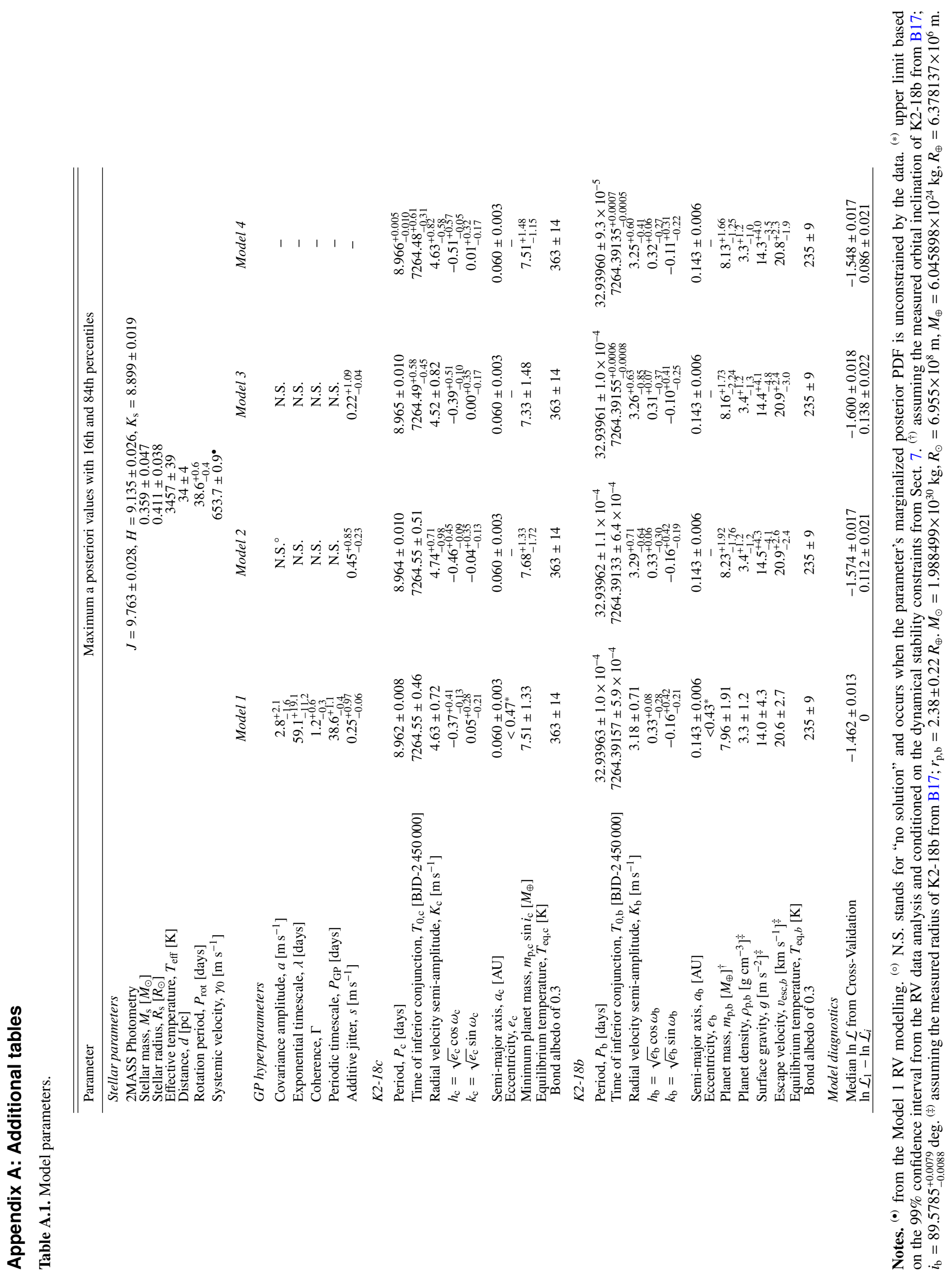


Table A.2. HARPS time series.

\begin{tabular}{|c|c|c|c|c|c|c|}
\hline BJD-2 450000 & $\begin{array}{c}\mathrm{RV} \\
{\left[\mathrm{m} \mathrm{s}^{-1}\right]}\end{array}$ & $\begin{array}{c}\sigma \mathrm{RV} \\
{\left[\mathrm{m} \mathrm{s}^{-1}\right]}\end{array}$ & $S$ index & $\mathrm{H} \alpha$ & FWHM & BIS \\
\hline 7117.565870 & 658.15 & 4.30 & 0.551 & 0.06337 & 3.050 & 9.397 \\
\hline 7146.526948 & 654.90 & 2.94 & 0.851 & 0.06646 & 3.068 & -3.008 \\
\hline 7146.646070 & 660.66 & 3.98 & 0.537 & 0.06850 & 3.079 & -6.150 \\
\hline 7148.518851 & 649.21 & 4.62 & 0.973 & 0.06667 & 3.071 & 8.583 \\
\hline 7199.503915 & 656.11 & 3.43 & 0.558 & 0.06597 & 3.090 & 16.922 \\
\hline 7200.503114 & 656.48 & 2.79 & 0.290 & 0.06625 & 3.080 & 19.343 \\
\hline 7204.491167 & 648.52 & 4.38 & 0.368 & 0.06409 & 3.076 & -10.068 \\
\hline 7390.845075 & 655.54 & 2.65 & 1.040 & 0.06721 & 3.106 & -0.196 \\
\hline 7401.779223 & 649.19 & 2.66 & 0.960 & 0.06640 & 3.105 & 1.064 \\
\hline 7403.826871 & 651.38 & 2.87 & 1.287 & 0.06598 & 3.106 & 5.332 \\
\hline 7404.814521 & 654.49 & 3.78 & 1.410 & 0.06679 & 3.095 & 6.633 \\
\hline 7405.789149 & 655.54 & 2.71 & 1.066 & 0.06644 & 3.106 & 7.934 \\
\hline 7407.773473 & 652.66 & 4.36 & - & 0.06680 & 3.048 & 0.311 \\
\hline 7410.791609 & 651.99 & 4.35 & 1.296 & 0.07166 & 3.114 & -2.344 \\
\hline 7412.810195 & 661.94 & 3.50 & 0.677 & 0.06618 & 3.094 & -8.771 \\
\hline 7417.787334 & 649.38 & 3.41 & 0.724 & 0.06615 & 3.092 & 7.447 \\
\hline 7418.799229 & 648.38 & 3.67 & 1.402 & 0.06842 & 3.100 & 2.274 \\
\hline 7420.791577 & 658.68 & 2.54 & 1.170 & 0.06738 & 3.099 & 11.285 \\
\hline 7421.794046 & 658.62 & 2.30 & 1.509 & 0.07463 & 3.099 & 11.310 \\
\hline 7422.781258 & 657.47 & 2.66 & 0.986 & 0.06505 & 3.104 & 7.032 \\
\hline 7424.777426 & 658.79 & 2.86 & 0.924 & 0.06642 & 3.104 & 5.314 \\
\hline 7425.850669 & 652.07 & 2.81 & 0.871 & 0.06636 & 3.088 & -0.471 \\
\hline 7446.704487 & 652.60 & 2.67 & 0.933 & 0.06481 & 3.108 & 1.511 \\
\hline 7447.830725 & 659.17 & 3.87 & 0.862 & 0.06539 & 3.085 & 0.282 \\
\hline 7448.686909 & 656.59 & 3.26 & 0.713 & 0.06470 & 3.098 & 0.282 \\
\hline 7450.675147 & 660.91 & 3.33 & 0.917 & 0.06521 & 3.102 & -8.218 \\
\hline 7451.677499 & 655.48 & 2.70 & 1.063 & 0.06575 & 3.111 & -5.083 \\
\hline 7452.695705 & 663.48 & 2.75 & 1.112 & 0.06532 & 3.093 & 1.667 \\
\hline 7453.701988 & 655.18 & 2.38 & 1.038 & 0.06689 & 3.104 & 10.356 \\
\hline 7456.704230 & 658.87 & 3.31 & 0.887 & 0.06586 & 3.111 & -17.835 \\
\hline 7457.683261 & 658.28 & 3.81 & 1.010 & 0.06615 & 3.101 & 12.792 \\
\hline 7458.660021 & 656.62 & 3.47 & 1.404 & 0.06634 & 3.099 & 9.160 \\
\hline 7472.784787 & 641.61 & 3.12 & 1.058 & 0.06653 & 3.094 & -1.001 \\
\hline 7473.684129 & 648.26 & 3.08 & 0.908 & 0.06529 & 3.096 & -1.293 \\
\hline 7474.737446 & 645.84 & 3.90 & 0.783 & 0.06621 & 3.085 & -6.991 \\
\hline 7475.698658 & 655.63 & 3.94 & 0.331 & 0.06647 & 3.094 & -0.455 \\
\hline 7476.707703 & 652.59 & 3.26 & 0.983 & 0.06517 & 3.091 & -0.985 \\
\hline 7477.674398 & 657.56 & 3.37 & 0.816 & 0.06554 & 3.100 & -4.990 \\
\hline 7478.631994 & 652.31 & 4.64 & 0.682 & 0.06713 & 3.093 & -9.857 \\
\hline 7479.737617 & 649.94 & 3.17 & 1.116 & 0.06541 & 3.086 & -9.996 \\
\hline 7486.661319 & 666.10 & 3.24 & 1.168 & 0.06527 & 3.096 & -10.681 \\
\hline 7487.617699 & 667.97 & 4.55 & 0.573 & 0.06504 & 3.091 & -0.986 \\
\hline 7488.670507 & 656.68 & 3.56 & 0.922 & 0.06431 & 3.086 & -8.201 \\
\hline 7567.516862 & 659.76 & 4.00 & 0.993 & 0.06530 & 3.096 & 10.014 \\
\hline 7576.473152 & 657.32 & 4.27 & 0.989 & 0.06587 & 3.115 & 7.131 \\
\hline 7584.477527 & 657.94 & 5.55 & 0.725 & 0.06563 & 3.080 & 16.445 \\
\hline 7786.842858 & 645.83 & 3.49 & 1.146 & 0.06430 & 3.093 & -1.668 \\
\hline 7787.825672 & 658.25 & 4.66 & 0.944 & 0.06581 & 3.092 & 5.481 \\
\hline 7790.828228 & 655.56 & 3.49 & 1.092 & 0.06500 & 3.088 & 1.878 \\
\hline 7791.843445 & 653.87 & 3.70 & 0.700 & 0.06414 & 3.093 & -3.719 \\
\hline 7792.815105 & 653.38 & 3.47 & 0.893 & 0.06861 & 3.083 & 6.408 \\
\hline 7801.827514 & 652.57 & 3.18 & 0.812 & 0.06518 & 3.094 & 12.389 \\
\hline 7802.790293 & 648.98 & 3.14 & 0.860 & 0.06504 & 3.094 & -3.525 \\
\hline 7803.809311 & 649.61 & 3.27 & 0.926 & 0.06616 & 3.091 & 18.332 \\
\hline 7810.806284 & 654.41 & 4.71 & 0.905 & 0.06593 & 3.098 & 6.496 \\
\hline 7814.760772 & 652.53 & 4.35 & - & 0.06632 & 3.098 & -0.563 \\
\hline 7815.759421 & 656.22 & 5.06 & - & 0.06536 & 3.086 & 15.990 \\
\hline 7817.748614 & 665.63 & 2.89 & 0.998 & 0.06423 & 3.103 & 7.162 \\
\hline 7830.668729 & 647.15 & 3.18 & 0.866 & 0.06476 & 3.084 & -1.992 \\
\hline 7832.659387 & 647.89 & 4.31 & 1.029 & 0.06292 & 3.079 & 14.048 \\
\hline 7834.636450 & 656.64 & 4.31 & 1.025 & 0.06407 & 3.091 & 9.870 \\
\hline 7835.596293 & 654.97 & 4.36 & 1.004 & 0.06308 & 3.093 & 11.521 \\
\hline 7836.626075 & 656.46 & 4.71 & 0.792 & 0.06491 & 3.093 & -2.004 \\
\hline 7839.650934 & 644.07 & 3.74 & 0.774 & 0.06437 & 3.076 & 5.253 \\
\hline 7841.638147 & 648.49 & 3.92 & 0.810 & 0.06487 & 3.089 & 0.543 \\
\hline 7843.648062 & 654.65 & 3.92 & 0.408 & 0.06404 & 3.090 & 12.825 \\
\hline 7844.626814 & 659.07 & 3.85 & 0.722 & 0.06417 & 3.087 & 1.584 \\
\hline 7846.692642 & 655.96 & 3.75 & 0.923 & 0.06435 & 3.086 & 5.210 \\
\hline 7847.693240 & 651.46 & 3.77 & 0.903 & 0.06363 & 3.094 & 8.538 \\
\hline 7848.677241 & 655.24 & 3.82 & 0.848 & 0.06445 & 3.102 & 1.280 \\
\hline 7849.696944 & 653.92 & 3.34 & 0.853 & 0.06440 & 3.082 & 9.616 \\
\hline 7872.656844 & 653.72 & 3.92 & 0.913 & 0.06371 & 3.082 & -5.325 \\
\hline 7873.525484 & 652.07 & 4.95 & 0.813 & 0.06478 & 3.094 & 13.489 \\
\hline 7874.671695 & 650.86 & 4.80 & 0.886 & 0.06543 & 3.088 & 1.857 \\
\hline 7875.596914 & 643.77 & 3.69 & 0.913 & 0.06467 & 3.077 & 5.739 \\
\hline
\end{tabular}

